\title{
THE ANTIMAXIMUM PRINCIPLE AND THE EXISTENCE OF A SOLUTION FOR THE GENERALIZED $p$-LAPLACE EQUATIONS WITH INDEFINITE WEIGHT
}

\author{
MIEKO TANAKA
}

Abstract. This paper treats the antimaximum principle and the existence of a solution for quasilinear elliptic equation $-\operatorname{div}(a(x,|\nabla u|) \nabla u)=\lambda m(x)|u|^{p^{-2}} u+h(x)$ in $\Omega$ under the Neumann boundary condition. Here, a map $a(x,|y|) y$ on $\bar{\Omega} \times \mathbb{R}^{N}$ is strictly monotone in the second variable and satisfies certain regularity conditions. This equation contains the $p$-Laplacian problem as a special case.

Mathematics subject classification (2010): 35J62, 35P30, 58E05.

Keywords and phrases: quasilinear elliptic equations, antimaximum principle, indefinite weight, nonlinear eigenvalue problems, the $p$-Laplacian, mountain pass theorem.

\section{REFERENCES}

[1] A. Ambrosetti, G. Prodi, A Primer of Nonlinear Analysis, Cambridge Studies Advanced Mathematics, 34, Cambridge University Press, Cambridge, 1995.

[2] A. Anane, O. Chakrone, N. Moradi, Maximum and anti-maximum principles for the pLaplacian with a nonlinear boundary condition, Electr. J. Differential Equations, Conference 14 (2006), 95-107.

[3] D. Arcoya, J. D. Rossi, Antimaximum principle for quasilinear problems, Adv. Differential Equations, 9 (2004), 1185-1200.

[4] M. Arias, J. Campos, M. Cuesta, J.-P. Gossez, An asymmetric Neumann problem with weights, Ann. Inst. Henri Poincaré., 25 (2008), 267-280.

[5] M. ARias, J. CAmpos, J.-P.GosseZ, On the antimaximum principle and the Fučik spectrum for the Neumann p-Laplacian, Differential Integral Equations, 13 (2000), 217-226.

[6] I. BIRINDELLI, Hopf's lemma and antimaximum principle in general domains, J. Differential Equations, 119 (1995), 450-472.

[7] E. Casas, L. A. Fernandez, A Green's formula for quasilinear elliptic operators, J. Math. Anal. Appl., 142 (1989), 62-73.

[8] P. Clement, L. Peletier, An antimaximum principle for second-order elliptic operators, J. Differential Equations, 34 (1979), 218-229.

[9] L. DAMASCELli, Comparison theorems for some quasilinear degenerate elliptic operators and applications to symmetry and monotonicity results, Ann. Inst. Henri Poincaré, 15 (1998), 493-516.

[10] J. Fleckinger, J.-P. Gossez, P. TAKaČ, F. DE Thelin, Existence, non existence et principle de l'antimaximum pour le p-Laplacien, C. R. Acad. Sci. Paris, 321 (1995), 731-734.

[11] J. Fleckinger, J.-P. Gossez, F. DE Thelin, Maximum and antimaximum principles: beyond the first eigenvalue, Differential Integral Equations, 22 (2009), 815-828.

[12] L. Gasińs KI, N. S. PAPAgeorgiou, Nonlinear Analysis, vol. 9, Chapman \& Hall/CRC, Boca Raton, Florida, 2006.

[13] T. Godoy, J.-P. Gossez, S. PaczKa, Antimaximum principle for elliptic problems with weight, Electr. J. Differential Equations, 1999 (1999), 1-15. 
[14] T. Godoy, J.-P. Gossez, S. PaCzKa, On the antimaximum principle for the p-Laplacian with indefinite weight, Nonlinear Anal., 51 (2002), 449-467.

[15] S. E. HABIB, N. Tsouli, On the spectrum of the p-Laplacian operator for Neumann eigenvalue problems with weights, Electr. J. Differential Equations, 2005 (2005), 181-190.

[16] P. HESs, An anti-maximum principle for linear elliptic equations with indefinite weight function, J. Differential Equations, 41 (1981), 369-374.

[17] G. M. Lieberman, Boundary regularity for solutions of degenerate elliptic equations, Nonlinear Anal., 12 (1988), 1203-1219.

[18] J. Mawhin, M. Willem, Critical Point Theory and Hamiltonian System, Springer-Verlag, New York, 1989.

[19] S. Miyajima, D. Motreanu, M. Tanaka, Multiple existence results of solutions for the Neumann problems via super- and sub-solutions, J. Funct. Anal., 262 (2012), 1921-1953.

[20] D. Motreanu, V. V. Motreanu, N. S. Papageorgiou, Multiple constant sign and nodal solutions for Nonlinear Neumann eigenvalue problems, Ann. Sc. Norm. Super. Pisa Cl. Sci. (5), 10 (2011), 729-755.

[21] D. Motreanu, N. S. Papageorgiou, Multiple solutions for nonlinear Neumann problems driven by a nonhomogeneous differential operator, Proc. Amer. Math. Soc., 139 (2011), 3527-3535.

[22] N. Stavrakakis, F. De Thelin, Principal eigenvalues and antimaximum principle for some quasilinear elliptic equations on $\mathbb{R}^{N}$, Math. Nachr., 212 (2000), 155-171.

[23] M. TANAKA, Existence results for quasilinear elliptic equations with indefinite weight, to appear in Abstract and Applied Analysis. 